\title{
Automotive Battery Equalizers Based on Joint Switched-Capacitor and Buck-Boost Converters
}

\author{
Kailong Liu, Member, IEEE, Zhile Yang, Member, IEEE, Xiaopeng Tang and Wenping Cao, Senior Member, \\ IEEE
}

\begin{abstract}
A series of integrated equalizers based on joint buckboost (BB) and switched-capacitor (SC) converters are proposed for balancing the voltages of series-connected battery packs. All these equalizers realize the any-cells-to-any-cells (AC2AC) equalization mode without increasing any MOSFETs and drivers. Corresponding operational principles are analyzed and the expressions of balancing currents are verified by experimental waveforms. According to the comparative balancing experiments for four and six series-connected Li-ion cells, one proposed CBB-PCSC equalizer, which achieves the dual AC2AC balancing modes through the integration of both coupled buck-boost (CBB) and parallel-connected switched-capacitor (PCSC) converters, leads to the highest balancing speed and efficiency. Moreover, compared with several conventional equalizers, this CBB-PCSC topology also has the compact size and low cost, making it become a well-performing integrated topology for automotive battery voltages equalization.
\end{abstract}

Index Terms-Li-ion batteries management, voltage equalization, any-cells-to-any-cells mode, electric vehicles.

\section{INTRODUCTION}

Lithium-ion (Li-ion) batteries are generally connected in series or in parallel to provide enough voltage and power for electric vehicles (EVs) [1]-[4]. One key but challenging issue is to equalize the inconsistent voltages among cells, owing to that the performance and safety of battery packs/modules are strongly related to the voltage consistency level [5]. In actual applications, slight differences in cell capacity, impedance or self-discharge rate may occur [6]. These differences tend to increase over time after charging/discharging cycles, further resulting in the reduced available capacity, accelerated health degradation and even safety problems for battery pack [7][10]. In this regard, it is vital to develop effective equalization topologies for battery pack to ensure that each cell can be operated within the satisfactory consistency level [11 12].

To date, various battery equalization approaches have been proposed in the literature, which can be mainly divided into passive equalizers and active equalizers [13]-[15]. Due to the

Manuscript received ...; revised ...; and accepted .... This work was supported by Royal Society Wolfson Research Merit Award (NoTEMP); Horizon 2020, RISE project No. 872001; the Faraday Institution funded Nextrode project (EP/T012412/1) and the Hong Kong Research Grant Council (16207717). (Corresponding authors: Kailong Liu and Xiaopeng Tang.)

$\mathrm{K}$. Liu is with the WMG, The University of Warwick, United Kingdom (kliu02@qub.ac.uk, kailong.liu@warwick.ac.uk).

Z. Yang is with the Shenzhen Institute of Advanced Technology, Chinese Academy of Sciences, 518055, China (zl.yang@siat.ac.cn).

$\mathrm{X}$. Tang is with the Hong Kong University of Science and Technology, Clear Water Bay, Kowloon, Hong Kong SAR. (xtangai@connect.ust.hk).

W. Cao is with the Aston University, Birmingham, United Kingdom.(w.p.cao@aston.ac.uk). simple structure with only one resistor and switch for each cell, the passive equalizer can be easily implemented in EVs [13]. However, this solution is limited as large energy dissipation and long equalization time. Besides, by using passive equalization, considerable heat will be generated, which leads to many thermal issues. To overcome these drawbacks, equalizers based on the active balancing have been designed. For active balancing, a proper active equalizer consisting of several energy storage components (i.e., capacitor, and inductor) is utilized to control power flow, further leading to an efficiencyconscious way to equalize battery cells [13 16].

According to the types of energy flux, active equalizer can be further classified into five topology categories: adjacent cell-to-cell (AC2C) [17]-[19], direct cell-to-cell (DC2C) [20][23], cell-to-pack (C2P) [24]-[27], pack-to-cell (P2C) [27 28], and any-cells-to-any-cells (AC2AC) [29 30]. The AC2C equalizer owns a simple structure with compact size, low cost, and small voltage stress on components [17]-[19]. However, the energy for such case is only transferred from one cell to adjacent cell, resulting in a relatively slow equalization speed and low efficiency for the multi-cell string. The DC2C equalizer is capable of directly transferring energy among arbitrary two cells regardless of their positions in the battery string [20][23]. However, just two cells can be equalized simultaneously by $\mathrm{DC} 2 \mathrm{C}$, leading to a slow equalization speed for a large battery pack. For example, Shang et al. [31] proposed a compact DC2C equalizer based on LC resonant and boost converters. As a result, a high balancing efficiency of $98 \%$ is achieved. The boost converter is employed to improve the voltage gap and balancing current, which provides a condition for zero-voltage gap (ZVG) between cells. By using the C2P equalizer or $\mathrm{P} 2 \mathrm{C}$ equalizer, energy can be transferred among individual cell and whole battery pack, further improving the equalization speed [24]-[28]. However, their corresponding step-up and step-down conversion ratios are extremely high or low for a large battery string, leading to the low conversion efficiency and large voltage stress on power switches. In comparison with the aforementioned equalizers that only achieve the 'time-shared' equalization, the AC2AC equalizer automatically transfers energy from all higher-voltage cells to all lower-voltage cells [2930]. Consequently, both the equalization speed and efficiency can be guaranteed, prompting the $\mathrm{AC} 2 \mathrm{AC}$ equalizer to become a promising way for equalizing inconsistency among cell voltages. It is worth mentioning that Shang et al. [30 32] designed a series of high-efficiency compact $\mathrm{AC} 2 \mathrm{AC}$ equalizers with simple control. By designing new switched-capacitor or forward-flyback converters, these equalizers can automatically and directly deliver energy from 
any higher voltage cells to lower voltage ones without the need of cell monitoring circuits, leading to a higher balancing speed independent of the cell number and the initial cell voltages.

In addition, several conventional topologies have been widely used for battery equalization. As shown in Fig. 1 (a), a classic active equalization architecture is developed by employing a BB converter for every two adjacent cells [17], bringing the benefits that the automatic and simultaneous equalization process can be achieved with a simple control implementation and low voltage stress on MOSFETs. Unfortunately, a large amount of MOSFETs and inductors are involved in this BB based equalizer, further leading to a large topology size and high cost. Moreover, the corresponding equalization speed and efficiency would be severely limited because the energy in this architecture can only be transferred from one cell to the adjacent one, i.e., the AC2C equalization [33].

Fig. 11 (b) shows the series-connected switched-capacitor (SCSC) equalizer for $n$ cells, where one capacitor is set for every two adjacent cells [18]. By switching the parallel capacitors back and forth repeatedly, the SCSC equalizer brings the cell voltages to average value [18]. However, energy is also transferred from one cell to the adjacent one, resulting in a severe penalty on the balancing efficiency and speed.

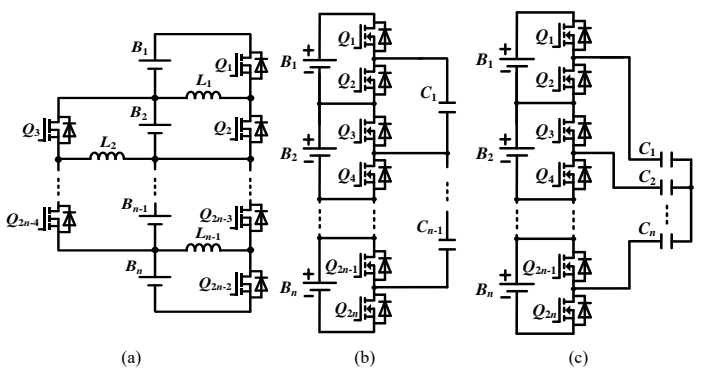

Fig. 1. The conventional equalizers. (a) BB topology [16]. (b) SCSC topology [17]. (c) PCSC topology [29].

Analogously, a parallel-connected switched-capacitor (PCSC) equalizer [29] with faster equalization speed and higher efficiency is presented in Fig. 1(c), which is capable of transferring energy from all higher-voltage cells to all lower-voltage cells, i.e., the AC2AC equalization, without considering the initial imbalanced statuses of cell voltages [29]. However, as illustrated in Fig. 11(c), each cell involves two MOSFETs and drivers, resulting in a large topology size with high cost. In this regard, complicated structure becomes a main bottleneck for large-scale industrial applications of the PCSC equalizer.

Fortunately, both BB and SC equalizers have the same halfbridge structure, generating the novel possibilities to integrate them into one topology. Specifically, we can integrate the SC equalizers into the $\mathrm{BB}$ equalizer, which not only uses less MOSFETs and isolated drivers, reducing circuit size and cost, but also achieves more balancing paths among cells, further increasing the equalization speed and efficiency.

Based upon the above discussion, this paper proposes several integrated equalizers based on joint $\mathrm{BB}$ and SC converters, which not only realize the simultaneous and efficient equalization for series-connected cells, but also achieve the improved AC2AC mode without using extra MOSFETs and drivers. Specifically, four key original contributions are made in this work. Firstly, a novel framework by integrating the inductor-based and SC-based equalizers into one topology is proposed, which will be helpful for the future improvement of battery equalization. Secondly, three integrated equalization topologies are designed to suit different applications and requirements. Thirdly, the analytical expressions of the balancing currents are derived and verified by experiments, which provides a guideline for the optimal design of the proposed equalizer. Finally, a comparison of the balancing results of the proposed equalizers is conducted through experimental tests, evaluating the performance of each integrated equalizer. Obviously, with dual AC2AC modes, the proposed CBB-PCSC equalizer outperforms other counterparts.

\section{INTEGRATED EQUALIZER AND OPERATION PRINCIPLES}

\section{A. Circuit Configuration and Operational Principles}

Driven by the idea of combining the benefits of both $\mathrm{BB}$ and SC converters, three integrated equalizers are designed, as shown in Fig. 2. From Fig. 2 (a), the interleaved buck-boost (IBB) converter and the PCSC converter are easily integrated into one topology due to the similar half-bridge structure. As a result, this integrated IBB-PCSC equalizer can not only achieve reliable $\mathrm{AC} 2 \mathrm{C}$ equalization between neighboring cells through the IBB converters but also realize the AC2AC equalization among the two-cell groups (e.g., $B_{1}-B_{2}, B_{3}$ $B_{4}, \ldots$ ) through the PCSC converter without increasing the numbers of the MOSFETs and drivers. This method has the obvious advantage of easy extension for odd and even cell numbers.

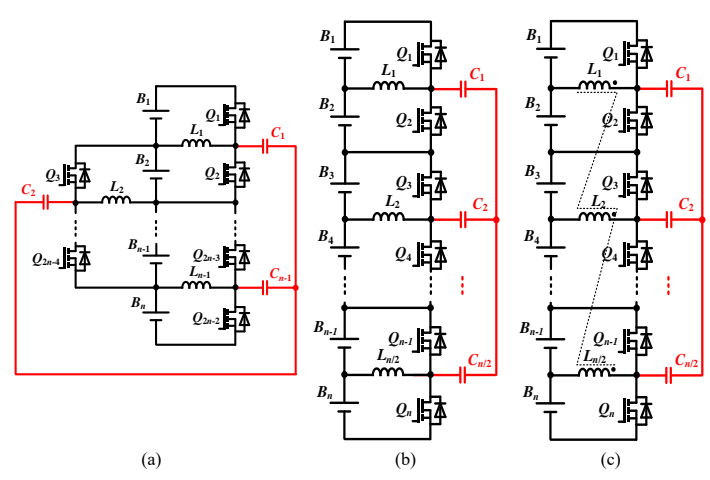

Fig. 2. The proposed integrated equalizers. (a) IBB-PCSC topology. (b) SBBPCSC topology. (c) CBB-PCSC topology.

However, one obvious drawback for the IBB-PCSC equalizer is that several BB converters (i.e., the left ones) share the repeated equalization functions with the PCSC converter, which not only increases energy loss but also leads to relatively large size and high cost. To further simplify the equalizer topology, a sequential buck-boost (SBB)-PCSC equalizer is proposed by only employing the relevant $\mathrm{BB}$ converters, as shown in Fig. 2 (b). Consequently, both the topology size and the corresponding cost are reduced. Besides, the SBB and PCSC converters could complement each other. Specifically, the equalization within every two-cell group can be realized 
by the SBB converter, while the $\mathrm{AC} 2 \mathrm{AC}$ equalization among all two-cell groups can be achieved by the PCSC converter.

To further improve the equalization performance, an integrated equalizer based on the coupled buck-boost (CBB) and the PCSC converters is proposed and shown in Fig. 2 (c). This CBB part is capable of realizing the effective AC2AC equalization for the series-connected battery string because the inductors here are also coupled in a multi-winding transformer. Through adopting double AC2AC equalizations, the speed and efficiency could be enhanced without using additional components. In comparison with the other two proposed equalizers, the topology structure of integrated CBB-PCSC equalizer is better in terms of low numbers of components to achieve best equalization for series connected cells.

\section{B. Operational Principles}

Taken the CBB-PCSC equalizer as an example, Fig. 3 shows the operational principles for six series-connected cells under the assumption of $V_{B 1}>V_{B 2}>\ldots>V_{B 6} . L_{1}, L_{2}$, and $L_{3}$ are the magnetic inductances of multi-winding transformer. $L_{L 1}, L_{L 2}$, and $L_{L 3}$ represent the leakage inductors of multiwinding transformer. $C_{1}, C_{2}$, and $C_{3}$ are the capacitors. $L_{C 1}$, $L_{C 2}$, and $L_{C 3}$ stand for the parasitic inductances in the PCSC equalizer. $R_{L 1}, R_{L 2}$, and $R_{L 3}$ are the equivalent resistances in the transformer windings. $R_{C 1}, R_{C 2}$, and $R_{C 3}$ represent the equivalent resistances in the capacitor branch circuits. This CBB-PCSC equalizer could be only controlled by a pair of complementary PWM signals for the odd and even MOSFETs, respectively, and has two steady working states in one switching period as:

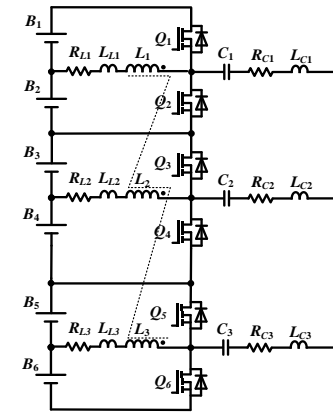

(a)

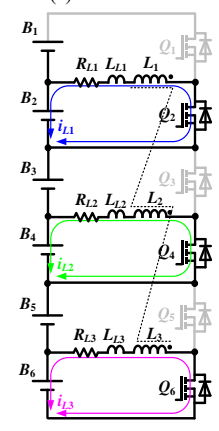

(c1)

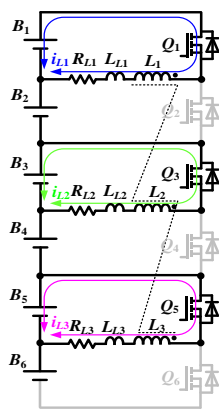

(b1)

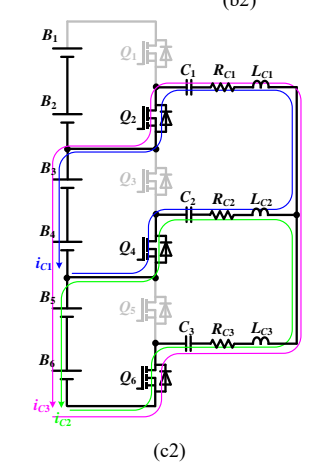

Fig. 3. Operational principles of the proposed CBB-PCSC equalizer under the assumption of $V_{B 1}>V_{B 2}>\ldots>V_{B 6}$. (a) Topology for CBB-PCSC. (b1), (b2) State I. (c1), (c2) State II.
State I $\left[\boldsymbol{t}_{\mathbf{0}}-\boldsymbol{t}_{\mathbf{1}}\right.$, Figs. 3 (b1), (b2)]: At $t_{0}$, the odd numbered MOSFETs $Q_{1}, Q_{3}, Q_{5}$ are turned $\mathrm{ON}$, and the even numbered MOSFETs $Q_{2}, Q_{4}, Q_{6}$ are turned OFF.

For State I, $L_{1}, L_{2}, L_{3}$ that charged during the last State II is connected to the odd cells $B_{1}, B_{3}, B_{5}$, respectively. Then the odd cells would be charged with an exponential decrease current due to $i_{L 1}, i_{L 2}, i_{L 3}$ cannot change suddenly, as shown in Fig. 3 (b1). As $V_{B 1}>V_{B 3}>V_{B 5}, i_{L 1}$ will first decrease to 0 , followed by $i_{L 2}$ and $i_{L 3}$. Then, $B_{1}, B_{3}, B_{5}$ start to charge $L_{1}, L_{2}, L_{3}$ with an exponential increase inductor current, respectively. Here the charging times meet $t_{B 1}<t_{B 3}<t_{B 5}$ and the discharging times meet $t_{B 1}>t_{B 3}>t_{B 5}$. As a result, $B_{1}$ releases more energy, $B_{3}$ releases less energy, and $B_{5}$ absorbs the energy from $B_{1}$ and $B_{3}$. Therefore, the equalization among $B_{1}, B_{3}, B_{5}$ can be achieved based on forward conversion. As shown in Fig. 3 (b2), the capacitors are connected in parallel with the corresponding upper cells $B_{1}-B_{4}$ through $Q_{1}, Q_{3}$, and $Q_{5}$. Three discharging paths are constructed from $B_{1}-B_{4}$ to $C_{1}-C_{3}$. Specifically, $i_{C 1}$ flows from $B_{1}-B_{2}$ to $C_{1}-C_{2}$ through $Q_{1}$ and $Q_{3}$. $i_{C 2}$ flows from $B_{3}-B_{4}$ to $C_{2}-C_{3}$ through $Q_{3}$ and $Q_{5} \cdot i_{C 3}$ flows from $B_{1}-B_{4}$ to $C_{1}$ and $C_{3}$ through $Q_{5}$ and $Q_{7}$. During this period, energy in the upper cells $B_{1}-B_{4}$ is transferred to the capacitors.

State II $\left[\boldsymbol{t}_{\mathbf{1}}-\boldsymbol{t}_{\mathbf{2}}\right.$, Figs. 3 (c1), (c2)]: The odd MOSFETs $Q_{1}$, $Q_{3}, Q_{5}$ keep being turned ON, and the even MOSFETs $Q_{2}$, $Q_{4}, Q_{6}$ keep being turned OFF.

For State II, $L_{1}, L_{2}, L_{3}$ are connected to the even cells $B_{2}$, $B_{4}, B_{6}$, respectively. Because $L_{1}, L_{2}, L_{3}$ have been charged during State I, $i_{L 1}, i_{L 2}, i_{L 3}$ will charge $B_{2}, B_{4}, B_{6}$ with an exponential decrease current, as shown in Fig. 3 (c1). Due to $V_{B 2}>V_{B 4}>V_{B 6}, i_{L 1}$ will first decrease to 0 , followed by $i_{L 2}$ and $i_{L 3}$. After that, $B_{2}, B_{4}, B_{6}$ start to charge $L_{1}, L_{2}$, $L_{3}$, respectively with an exponential increase inductor current. Here the charging times meet $t_{B 2}<t_{B 4}<t_{B 6}$ and the discharging times meet $t_{B 2}>t_{B 4}>t_{B 6}$. Consequently, $B_{2}$ releases more energy, $B_{4}$ releases less energy, and $B_{6}$ absorbs the energy from $B_{2}$ and $B_{4}$. In addition, during States I and II, energy is automatically transferred from the odd cells with higher voltages to the even cells with lower voltages based on flyback conversion. Therefore, the equalization between the odd and even cells is realized. As shown in Fig. 3 (c2), the capacitors are connected in parallel with the corresponding lower cells $B_{3}-B_{6}$ through $Q_{2}, Q_{4}$, and $Q_{6}$. Three charging paths to cells are constructed. Specifically, $i_{C 1}$ flows from $C_{1}$ $C_{2}$ to $B_{3}-B_{4}$ through $Q_{2}$ and $Q_{4}$, which achieves the energy transfer from $B_{1}-B_{2}$ to $B_{3}-B_{4}$. $i_{C 2}$ flows from $C_{2}-C_{3}$ to $B_{5^{-}}$ $B_{6}$ through $Q_{4}$ and $Q_{6}$, which achieves the energy transfer from $B_{3}-B_{4}$ to $B_{5}-B_{6}$. $i_{C 2}$ flows from $C_{1}$ and $C_{3}$ to $B_{3}-B_{6}$ through $Q_{2}$ and $Q_{6}$, which achieves the energy transfer from $B_{1}-B_{2}$ to $B_{5}-B_{6}$.

According to the above analysis, it is evident that both the $\mathrm{CBB}$ and PCSC converters can achieve the AC2AC equalization among the cells, benefitting the improvements of both equalization speed and efficiency.

\section{Analysis of the balancing currents}

This subsection analyzes the balancing currents of CBBPCSC equalizer. For the CBB part, based upon Kirchhoff's 
voltage law (KVL), the voltage across the magnetic inductance of the $\mathrm{CBB}$ is equal to the average voltage of cells as,

$$
V_{L m}=\frac{V_{B 1}+V_{B 2}+V_{B 3}+V_{B 4}+V_{B 5}+V_{B 6}}{6} .
$$

Besides, the winding currents of the $\mathrm{CBB}$ equalizer during the first half period $(0<t \leq 0.5 T)$ can be expressed as,

$$
\left\{\begin{array}{l}
L_{L 1} \frac{d i_{L 1}}{d t}+i_{L 1} R_{L 1}=\frac{5 V_{B 1}-V_{B 2}-V_{B 3}-V_{B 4}-V_{B 5}-V_{B 6}}{6} \\
L_{L 2} \frac{d i_{L 2}}{d t}+i_{L 2} R_{L 2}=\frac{5 V_{B 3}-V_{B 1}-V_{B 2}-V_{B 4}-V_{B 5}-V_{B 6}}{6} \\
L_{L 3} \frac{d i_{L 3}}{d t}+i_{L 3} R_{L 3}=\frac{5 V_{B 5}-V_{B 1}-V_{B 2}-V_{B 3}-V_{B 4}-V_{B 6}}{6}
\end{array}\right.
$$

Analogously, the windings currents during the second half period $(0.5 T<t \leq T)$ can be expressed as,

$$
\left\{\begin{array}{l}
L_{L 1} \frac{d i_{L 1}}{d t}+i_{L 1} R_{L 1}=\frac{V_{B 1}+V_{B 3}+V_{B 4}+V_{B 5}+V_{B 6}-5 V_{B 2}}{6} \\
L_{L 2} \frac{d i_{L 2}}{d t}+i_{L 2} R_{L 2}=\frac{V_{B 1}+V_{B 2}+V_{B 3}+V_{B 5}+V_{B 6}-5 V_{B 4}}{6} \\
L_{L 3} \frac{d i_{L 3}}{d t}+i_{L 3} R_{L 3}=\frac{V_{B 1}+V_{B 2}+V_{B 3}+V_{B 4}+V_{B 5}-5 V_{B 6}}{6}
\end{array}\right.
$$

According to (2) and (3), the winding currents of the CBB equalizer can be solved as (4) and (5) at the front of next page.

It is important to note that the winding current of the $\mathrm{CBB}$ equalizer is in inverse proportion to the equivalent resistance $R_{L}$. The larger the cell voltage difference, the larger the winding current. As a result, decreasing the switching frequency or the leakage inductance can improve the balancing current. The cell balancing current is independent of the magnetic inductance, while the leakage inductance only affects the balancing current instead of the final equalized cell voltage.

For the PCSC part, due to the parasitic inductance in circuits, the SC converter can be equivalent to a resonant LC converter. When the switching frequency is equal to the inherent resonant frequency of the LC converter, the LC converter achieves the maximum energy transfer during one switching period. Let the input and output of the resonant LC converter are a higher voltage $V_{H}$ and a lower voltage $V_{L}$ respectively, according to the KVL, there exists a nonlinear relation between $V_{H}$ and the resonance current $i_{L C}$ during the first half switching period as,

$$
V_{H}=v_{C}+L \cdot \frac{d i_{L C}}{d t}+R \cdot i_{L C}
$$

where $v_{C}$ is the capacitor voltage. $L$ means the inductance. $R$ stands for the equivalent resistance.

Besides, $i_{L C}$ is also dependent on $v_{C}$ and expressed as,

$$
i_{L C}=C \cdot \frac{d v_{C}}{d t}
$$

where $C$ represents the capacitance of the capacitor. Based upon (6) and (7), the sinusoidal current during half switching period can be described as,

$$
i_{L C}(t)=A \cdot \sin (\beta \cdot t+B) \cdot e^{\alpha t},
$$

where

$$
\begin{aligned}
\alpha & =-\frac{R}{2 L}, \\
\beta & =\sqrt{\frac{1}{L \cdot C}-\frac{R^{2}}{4 L^{2}}} .
\end{aligned}
$$

According to (10), the corresponding resonant frequency $f_{0}$ could be described as,

$$
f_{0}=\frac{\sqrt{\frac{1}{L \cdot C}-\frac{R^{2}}{4 L^{2}}}}{2 \pi} .
$$

Under the conditions of $t=0$ and $i_{L C}(0)=0$, (8) would be further deduced as $B=0$.

Combining (6) and (8), the capacitor voltage could be further described by,

$$
v_{C}(t)=V_{H}-A \cdot \beta \cdot L \cdot \cos (\beta t) \cdot e^{\alpha t}-\frac{A \cdot R}{2} \cdot \sin (\beta t) \cdot e^{\alpha t}
$$

At $t=0$ and $t=\pi / \beta$, the capacitor voltage satisfies,

$$
v_{C}(0)+v_{C}\left(\frac{2 \pi}{\beta}\right)=V_{H}+V_{L} .
$$

Combining (12) and (13), parameter $A$ could be deduced as,

$$
A=\frac{V_{H}-V_{L}}{\beta \cdot L \cdot\left(1-e^{\frac{\alpha}{\beta} \pi}\right)} .
$$

With the explicit expressions of $\alpha, \beta, A$, and $B$, the resonance current could be written as,

$$
i_{L C}(t) \approx \frac{2\left(V_{H}-V_{L}\right)}{\pi \cdot R} \cdot \sin \left(\sqrt{\frac{1}{L \cdot C}-\frac{R^{2}}{4 L^{2}}} \cdot t\right) \cdot e^{-\frac{R}{2 L} t}
$$

As shown in Fig. 3, in order to simplify the analysis, it is reasonably assumed that the capacitances, resistances, and parasitic inductances are equal. According to (15), the balancing currents of the PCSC equalizer can be expressed as,

$$
\left\{\begin{array}{l}
i_{C 1}(t)=\frac{V_{B 1}+V_{B 2}-V_{B 3}-V_{B 4}}{\pi \cdot R_{C}} \cdot \sin \left(\frac{1}{\sqrt{L_{C} \cdot C-\frac{R_{C}^{2}}{4 L_{C}^{2}}}} \cdot t\right) \cdot e^{\frac{-R_{C}}{2 L_{C}} \cdot t} \\
i_{C 2}(t)=\frac{V_{B 3}+V_{B 4}-V_{B 5}-V_{B 6}}{\pi \cdot R_{C}} \cdot \sin \left(\frac{1}{\sqrt{L_{C} \cdot C-\frac{R_{C}^{2}}{4 L_{C}^{2}}}} \cdot t\right) \cdot e^{\frac{-R_{C}}{2 L_{C}} \cdot t} \\
i_{C 3}(t)=\frac{V_{B 1}+V_{B 2}-V_{B 5}-V_{B 6}}{\pi \cdot R_{C}} \cdot \sin \left(\frac{1}{\sqrt{L_{C} \cdot C-\frac{R_{C}^{2}}{4 L_{C}^{2}}}} \cdot t\right) \cdot e^{\frac{-R_{C}}{2 L_{C}} \cdot t}
\end{array}\right.
$$

where $R_{C}, L_{C}$, and $C$ are the uniform equivalent resistance, parasitic inductance, and capacitance, respectively. The balancing current of the PCSC equalizer is directly proportional to the cell voltage difference. Decreasing $R_{C}$ or increasing $L_{C}$ can reduce the attenuation of the amplitude of the balancing current. In a real application, the parameters in (11) may change over time, leading to the small change of $f_{0}$. This change could cause the variations of balancing time but would not affect the final balanced battery voltage. In this context, the proposed balancing circuit is able to adapt to this change.

According to the above analyses, it can be seen that the balancing current of the $\mathrm{CBB}$ part decreases as the switching frequency increases, and vice versa. Nevertheless, the PCSC part has a tough requirement for the switching frequency. According to (16), when the switching frequency reduces from $f_{0}$, the balancing current of PCSC part will also decrease. However, when the switching frequency is higher than $f_{0}$, 


$$
\begin{gathered}
\left\{\begin{array}{l}
i_{L 1}(t)=\frac{5 V_{B 1}-V_{B 2}-V_{B 3}-V_{B 4}-V_{B 5}-V_{B 6}}{6 R_{L}}-\frac{2 V_{B 1}+2 V_{B 2}-V_{B 3}-V_{B 4}-V_{B 5}-V_{B 6}}{3 R_{L}} \cdot \frac{1}{e^{-\frac{R_{L}}{L_{L} \cdot \frac{T}{2}}+1}} \cdot e^{-\frac{R_{L}}{L_{L}} t} \\
i_{L 2}(t)=\frac{5 V_{B 3}-V_{B 1}-V_{B 2}-V_{B 4}-V_{B 5}-V_{B 6}}{6 R_{L}}-\frac{2 V_{B 3}+2 V_{B 4}-V_{B 1}-V_{B 2}-V_{B 5}-V_{B 6}}{3 R_{L}} \cdot \frac{1}{e^{-\frac{R_{L}}{L_{L}} \cdot \frac{T}{2}}+1} \cdot e^{-\frac{R_{L}}{L_{L}} t}, 0<t \leq \frac{T}{2} . \\
i_{L 3}(t)=\frac{5 V_{B 5}-V_{B 1}-V_{B 2}-V_{B 3}-V_{B 4}-V_{B 6}}{6 R_{L}}-\frac{2 V_{B 5}+2 V_{B 6}-V_{B 1}-V_{B 2}-V_{B 3}-V_{B 4}}{3 R_{L}} \cdot \frac{1}{e^{-\frac{R_{L}}{L_{f}} \cdot \frac{T}{2}}+1} \cdot e^{-\frac{R_{L}}{L_{f}} t}
\end{array}\right. \\
\left\{\begin{array}{l}
i_{L 1}(t)=\frac{V_{B 1}+V_{B 3}+V_{B 4}+V_{B 5}+V_{B 6}-5 V_{B 2}}{6 R_{L}}+\frac{2 V_{B 1}+2 V_{B 2}-V_{B 3}-V_{B 4}-V_{B 5}-V_{B 6}}{3 R_{L}} \cdot \frac{1}{e^{-\frac{R_{L}}{L_{L}} \cdot \frac{T}{2}}+1} \cdot e^{-\frac{R_{L}}{L_{L}}\left(t-\frac{T}{2}\right)} \\
i_{L 2}(t)=\frac{V_{B 1}+V_{B 2}+V_{B 3}+V_{B 5}+V_{B 6}-5 V_{B 4}}{6 R_{L}}+\frac{2 V_{B 3}+2 V_{B 4}-V_{B 1}-V_{B 2}-V_{B 5}-V_{B 6}}{3 R_{L}} \cdot \frac{1}{e^{-\frac{R_{L}}{L_{L}} \cdot \frac{T}{2}}+1} \cdot e^{-\frac{R_{L}}{L_{L}}\left(t-\frac{T}{2}\right)}, \frac{T}{2}<t \leq T . \\
i_{L 2}(t)=\frac{V_{B 1}+V_{B 2}+V_{B 3}+V_{B 4}+V_{B 5}-5 V_{B 6}}{6 R_{L}}+\frac{2 V_{B 5}+2 V_{B 6}-V_{B 1}-V_{B 2}-V_{B 3}-V_{B 4}}{3 R_{L}} \cdot 1 \\
i^{-\frac{R_{L}}{L_{L}}\left(t-\frac{T}{2}\right)}
\end{array}\right.
\end{gathered}
$$

the balancing current will charge the higher-voltage cell in reverse, leading to a lower average current and efficiency. Particularly, when the switching frequency is twice $f_{0}$, the balancing current will charge and discharge the higher-voltage cell during the first half switching period, and then charge and discharge the lower-voltage cell during the second half switching period. In this case, no energy is transferred from the higher-voltage cell to the lower-voltage cell. Therefore, the switching frequency is optimally set as the resonant frequency $f_{0}$ of the PCSC equalizer. In reality, the difference of equivalent resistances, inductances and capacitances would affect the amplitude of balancing current, further affecting the balancing time of designed circuit. However, the balanced battery voltage would not be affected when the switching frequency is within twice $f_{0}$. That is, the effectiveness of the balancing circuit can be also guaranteed.

\section{EXPERIMENTAL RESULTS}

In this section, two experimental prototypes were set up to equalize four and six series-connected Li-ion battery strings. Here, the nominal capacity of adopted Li-ion battery is 1100 mAh. 49W2871 MOSFETs are used for $Q_{1}-Q_{6}$. Detailed of the inductors, capacitors, and multi-winding transformer are illustrated in Table I. According to (11), with measured $L_{C}$ $0.6 \mathrm{uH}, f_{0}$ is approximatively calculated as $50 \mathrm{kHz}$.

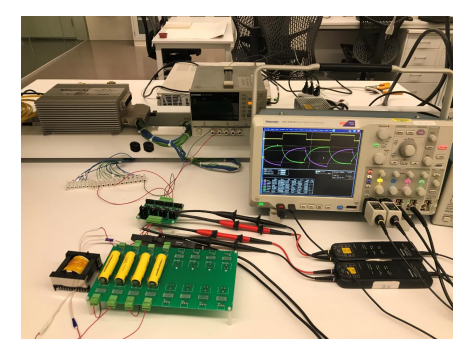

Fig. 4. Hardware setups of the balancing circuits.

Fig. 4 shows the hardware setups of the balancing circuits, while Fig. 5 details the corresponding experimental waveforms of the balancing currents for the CBB-PCSC equalizer. From Fig. 5 (a), it is seen that the winding currents of the CBB are linear waveforms, indicating that the balancing currents are mainly affected by the leakage inductances $L_{L 1}-L_{L 3}$ rather

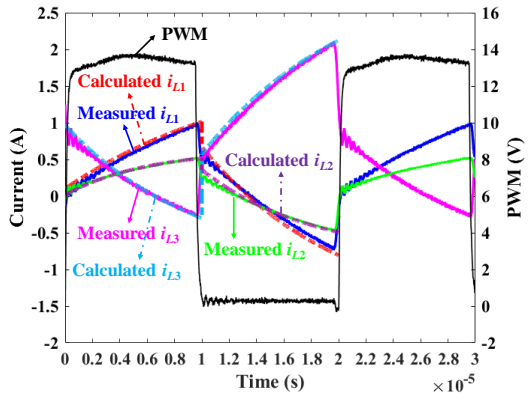

(a)

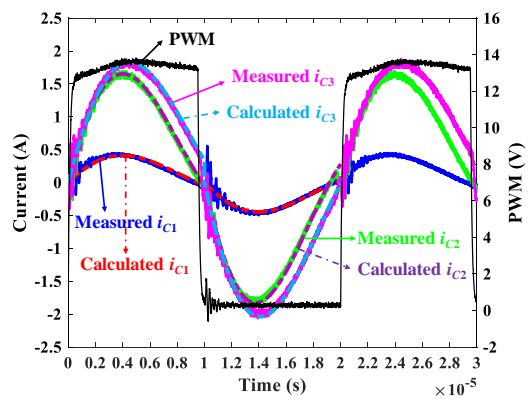

(b)

Fig. 5. Experimental waveforms of balancing currents of CBB-PCSC equalizer at $50 \mathrm{kHz}$. (a) The winding currents. (b) The capacitor currents.

than the magnetic inductances $L_{m 1}-L_{m 3}$. Here the maximum amplitude of the winding current (i.e., $i_{L 3}$ ), reaches $2 \mathrm{~A}$.

For the capacitor currents, the approximately sine waveforms are generated at $50 \mathrm{kHz}$ due to the effects of the parasitic inductances in circuits, resulting in a fully-charged or fullydischarged condition for the capacitors, as shown in Fig. 5(b). Consequently, CBB-PCSC equalizer can achieve maximum energy transfer during a switching cycle, further leading to a potential high efficiency. Here the maximum amplitude of the capacitor current, i.e., $i_{C 3}$, reaches $2 \mathrm{~A}$.

Next, in order to investigate the effectiveness of the proposed integrated equalizer, the conventional $\mathrm{BB}$ equalizer [17] is first used as a comparison to equalize four seriesconnected Li-ion cells $\left(B_{1}, B_{2}, B_{3}\right.$, and $\left.B_{4}\right)$ with various initial voltages $(3.239 \mathrm{~V}, 3.195 \mathrm{~V}, 2.968 \mathrm{~V}$, and $2.430 \mathrm{~V}$ respectively). The corresponding experimental results in terms of cell voltage, balancing current and balancing efficiency 


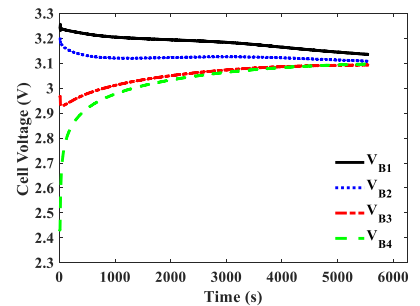

(a)

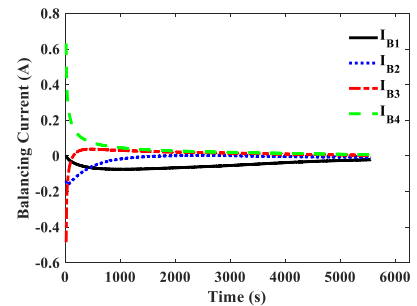

(b)

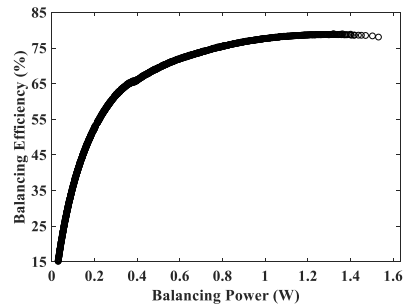

(c)

Fig. 6. Balancing results of the conventional BB equalizer [6] for four series-connected cells. (a) Cell voltage. (b) Balancing current. (c) Balancing efficiency.

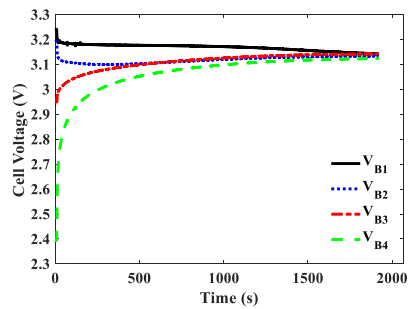

(a)

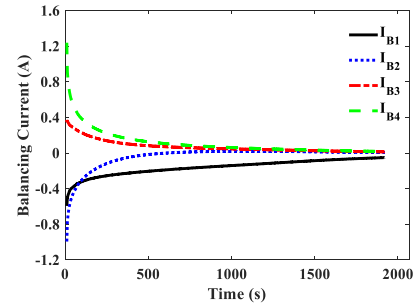

(b)

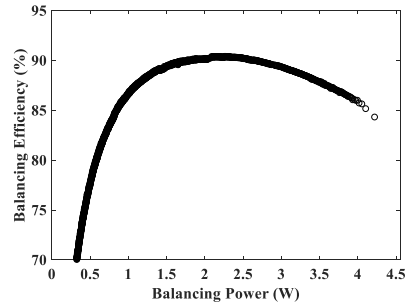

(c)

Fig. 7. Balancing results of the proposed IBB-PCSC equalizer for four series-connected cells. (a) Cell voltage. (b) Balancing current. (c) Balancing efficiency.

TABLE I

Parameters of the Inductors, CAPACITORS, AND Multi-WINDing Transformers

\begin{tabular}{|c|c|c|c|c|c|c|c|c|c|}
\hline Inductors & $L(\mu \mathrm{H})$ & $R_{L}(\mathrm{~m} \Omega)$ & Capacitors & $C(\mu \mathrm{F})$ & $R_{C}(\mathrm{~m} \Omega)$ & Windings of Transformer & $L_{m}(\mu \mathrm{H})$ & $L_{L}(\mu \mathrm{H})$ & $R_{L}(\mathrm{~m} \Omega)$ \\
\hline$L_{2}$ & 127.3 & 24 & $C_{2}$ & 13.8 & 47 & $W_{2}$ & 121.3 & 2.64 & 40 \\
\hline$L_{3}$ & 125.4 & 36 & $C_{3}$ & 13.6 & 50 & $W_{3}$ & 121.1 & 2.34 & 26 \\
\hline
\end{tabular}

evolutions are illustrated in Fig. 6(a), (b), and (c), respectively. Here, the maximum initial voltage gap is $0.809 \mathrm{~V}$ for $B_{1}$ and $B_{4}$. By using the conventional $\mathrm{BB}$ equalizer, this maximum voltage gap can only be reduced to $0.042 \mathrm{~V}$ after nearly $5500 \mathrm{~s}$, implying a slow equalization speed and poor equalization performance. Besides, due to just $\mathrm{AC} 2 \mathrm{C}$ equalization, the maximum balancing current can only reach $0.63 \mathrm{~A}$ for $B_{4}$. Fig. 6(c) shows the measured balancing efficiency versus the output power in this case. It is evident that the balancing efficiency is unsatisfactory with only the maximum value of $78.7 \%$ at $1.34 \mathrm{~W}$ balancing power.

Fig. 7 illustrates the experimental equalization results of the proposed integrated IBB-PCSC equalizer for four seriesconnected Li-ion cells. For comparison, the initial cell voltages are also set to $3.239 \mathrm{~V}, 3.195 \mathrm{~V}, 2.968 \mathrm{~V}$, and $2.430 \mathrm{~V}$, respectively. From Fig. 7(a), the maximum voltage gap reduces to only $0.02 \mathrm{~V}(97.5 \%$ decrease) after $1988 \mathrm{~s}$, which is $63.9 \%$ less than that of the conventional $\mathrm{BB}$ equalizer. Besides, here the maximum balancing current becomes $1.2 \mathrm{~A}$ for $B_{4}$, and the equalization efficiency is also increased to $90.4 \%$ at $2.17 \mathrm{~W}$ balancing power. These significant improvements are mainly caused by the AC2AC equalization of the PCSC. However, the BB converters still cause large energy loss, limiting the further increase of the balancing efficiency.

Fig. 8 illustrates the balancing results of the proposed SBBPCSC equalizer. It can be seen that the voltage gap between $B_{1}$ and $B_{4}$ reduces to $0.02 \mathrm{~V}$ after $1908 \mathrm{~s}$, which is quicker than that in the IBB-PCSC equalizer case. Nevertheless, the maximum efficiency value here is $92.6 \%$ at $1.768 \mathrm{~W}$ balancing power, indicating that removing the superfluous $\mathrm{BB}$ converters benefits the balancing efficiency. Therefore, we can conclude that the proposed SBB-PCSC equalizer has smaller size, lower cost, higher efficiency and speed than the IBB-PCSC equalizer.

Fig. 9 illustrates the equalization results of the proposed CBB-PCSC equalizer. Because the CBB and PCSC equalizers simultaneously achieve the AC2AC equalization, all the cells are balanced towards the average cell voltage. The highervoltage cells are balanced by discharging and the lower-voltage cells are balanced by charging. As shown in Fig. 9 (b), the higher the battery voltage difference, the greater the balancing current changes. It can be seen that by using the CBBPCSC equalizer, the total time is reduced to 1600 s, while the balancing current is increased to 1.3A. Particularly, the equalization efficiency is improved to $96 \%$. In conclusion, the proposed CBB-PCSC equalizer achieves the highest speed and efficiency among three integrated topologies.

In order to further identify the benefits of proposed SBBPCSC and CBB-PCSC equalizers, the balancing results of six series-connected cells under the same initial conditions for other equalizers including SBB equalizer and SBB-SCSC equalizer [19] are obtained and illustrated in Fig. 10] As shown in Fig. 10 (a), it seems that the SBB equalizer can only realize the equalization within each two-cell group, and is difficult to achieve the equalization among two-cell groups. From Fig. 10 (b), the proposed SBB-SCSC equalizer [19] achieves the full equalizations, as the SBB equalizer realizes 


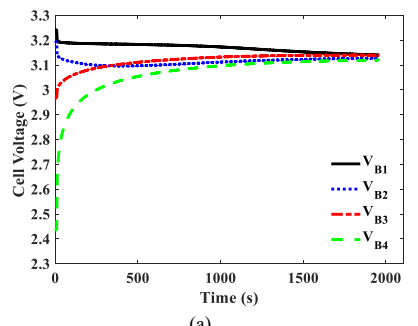

(a)

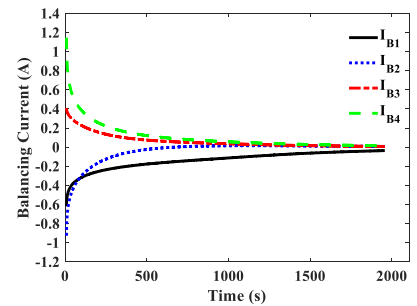

(b)

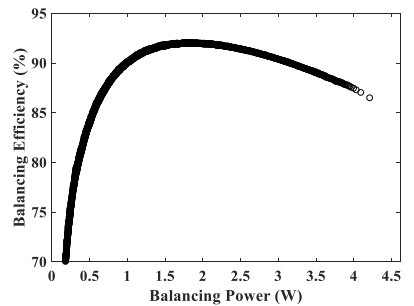

(c)

Fig. 8. Balancing results of the proposed SBB-PCSC equalizer for four series-connected cells. (a) Cell voltage. (b) Balancing current. (c) Balancing efficiency.

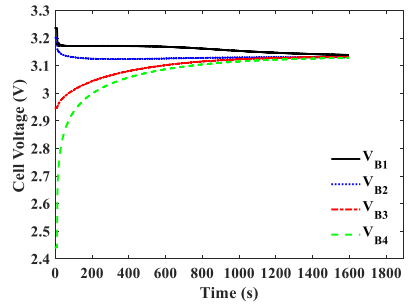

(a)

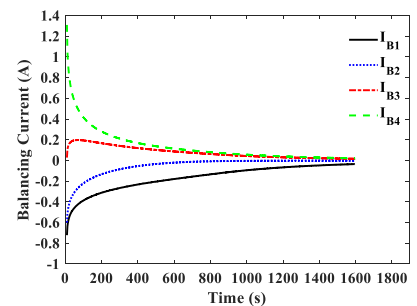

(b)

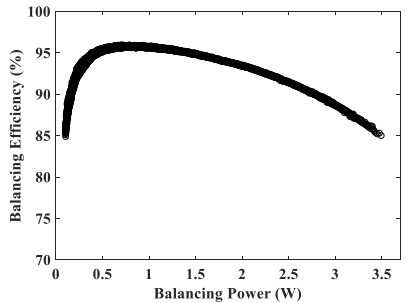

(c)

Fig. 9. Balancing results of the proposed CBB-PCSC equalizer for four series-connected cells. (a) Cell voltage. (b) Balancing current. (c) Balancing efficiency.

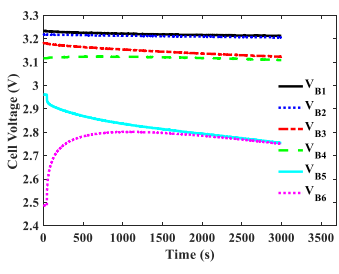

(a)

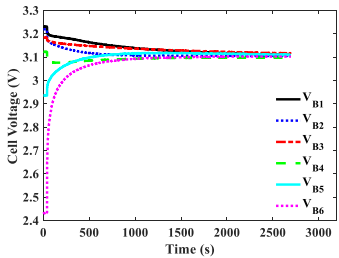

(c)

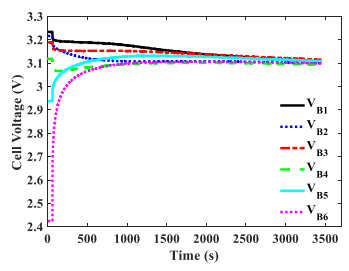

(b)

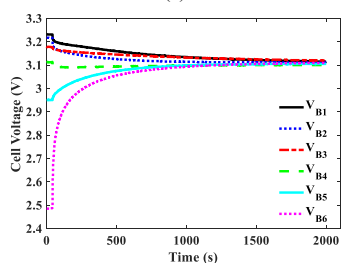

(d)
Fig. 10. A comparison of the balancing results of the proposed equalizers for six series-connected cells. (a) The SBB equalizer. (b) The SBB-SCSC equalizer [19]. (c) The SBB-PCSC equalizer. (d) The CBB-PCSC equalizer.

the $\mathrm{AC} 2 \mathrm{C}$ equalization within every two-cell group and the SCSC equalizer achieves the AC2C equalization between the two-cell groups. However, the balancing time is up to $3500 \mathrm{~s}$. By comparison, due to the achievement of AC2AC equalization among the two-cell groups, the balancing time of the SBB-PCSC equalizer is reduced to 2700s, as illustrated in Fig. 10 (c). From Fig. 10 (d), the balancing time of the CBB-PCSC equalizer is the smallest one (here is just 2000s) because of the AC2AC equalization for all cells. Besides, after balancing process, the maximum voltage difference among cells is less than $15 \mathrm{mV}$.

\section{COMParison With CONVENTIONAL EQUALIZERS}

In this section, to further evaluate the performance of proposed integrated equalizers, a comparison in terms of the component number, circuit size, total cost, efficiency, balancing mode, current, and speed is illustrated in Tables II.
The effectiveness of this comparison way has been proven in [34]. For this comparison, battery string consists of 8 battery modules with 12 cells in one module. Total cost is calculated through the quantity of the components and the component cost per unit. Circuit size is evaluated based on the component number and the volume of each component. Balancing speed is evaluated by the balancing paths and the balancing current. The BB equalizer [17] can achieve the automatic and simultaneous balancing between every two adjacent cells with a simple control. The main advantage is the modularization design and low voltage stress on switches. However, this equalizer needs 190 MOSFETs and 95 inductors, leading to a large size and high cost (\$213.8).

Through using two MOSFETs for one cell and one capacitor for two adjacent cells to realize the $\mathrm{AC} 2 \mathrm{C}$ equalization, the SCSC equalizer [18] still owns characteristics of large size, high cost (\$215.8), and small balancing current (0.57A). Moreover, as the cell number increases, the corresponding balancing efficiency will exponentially decrease.

As an improvement of the SCSC equalizer, the PCSC equalizer [29] achieves the AC2AC equalization, bringing the benefits that the balancing speed and efficiency are independent of cell number and initial imbalanced statuses of cell voltages. However, this PCSC equalizer still requires two MOSFETs for one cell, leading to a large size and high cost (\$216). In addition, the energy from the source cell must go through two capacitors before reaching the target cells, resulting in a small balancing current $(0.57 \mathrm{~A})$ and medium speed. The SBB-SCSC equalizer in [19] requires only one MOSFET for each cell, leading to a medium size and low cost (\$120). However, the SBB part only can realize the AC2C equalization within every two-cell group and the SCSC part only can achieve the AC2C equalization between the two-cell groups, resulting in a medium balancing speed.

Through simply integrating BB and PCSC equalizers, the IBB-PCSC equalizer presents the highest cost (\$225.8), which 
TABLE II

A COMPARISON OF BATTERY EQUALIZERS

\begin{tabular}{|c|c|c|c|c|c|c|c|c|c|c|c|}
\hline \multirow{2}{*}{$\begin{array}{l}\text { Equalizers } \\
\text { BB equalizer [17] }\end{array}$} & \multicolumn{4}{|c|}{ Component number } & \multirow{2}{*}{$\begin{array}{l}\text { Size } \\
\text { Large }\end{array}$} & \multirow{2}{*}{\multicolumn{2}{|c|}{ Cost $(\$)^{\mathrm{a}}$}} & \multirow{2}{*}{$\begin{array}{c}\text { Efficiency } \\
78.70 \%\end{array}$} & \multirow{2}{*}{$\begin{array}{c}\text { Balancing mode } \\
\mathrm{AC} 2 \mathrm{C}\end{array}$} & \multirow{2}{*}{$\begin{array}{c}\text { Current (A) } \\
0.63\end{array}$} & \multirow{2}{*}{$\begin{array}{c}\text { Speed } \\
\text { Low }\end{array}$} \\
\hline & $\mathrm{M}$ & $\mathrm{L}$ & $\mathrm{C}$ & $\mathrm{T}$ & & & & & & & \\
\hline SCSC equalizer [18] & 192 & 0 & 95 & 0 & Large & 215.8 & High & $93 \%$ & $\mathrm{AC} 2 \mathrm{C}$ & 0.57 & Low \\
\hline PCSC equalizer [29] & 192 & 0 & 96 & 0 & Large & 216 & High & $94 \%$ & $\mathrm{AC} 2 \mathrm{AC}$ & 0.57 & Medium \\
\hline IBB-PCSC equalizer & 190 & 95 & 48 & 0 & Large & 225.8 & High & $90.40 \%$ & $\mathrm{AC} 2 \mathrm{C}+\mathrm{AC} 2 \mathrm{AC}$ & 1.2 & Medium \\
\hline SBB-PCSC equalizer & 96 & 48 & 48 & 0 & Medium & 120 & Low & $92.60 \%$ & $\mathrm{AC} 2 \mathrm{C}+\mathrm{AC} 2 \mathrm{AC}$ & 1.1 & Medium \\
\hline Proposed CBB-PCSC equalizer & 96 & 0 & 52 & 9 & Small & 136 & Medium & $96 \%$ & $\mathrm{AC} 2 \mathrm{AC}+\mathrm{AC} 2 \mathrm{AC}$ & 1.3 & High \\
\hline
\end{tabular}

(T) (3) [24].

is caused by many involved components. However, this proposed equalizer also owns several benefits such as the easy extension for odd and even numbers of cell.

In fact, the SBB-PCSC equalizer is an improvement of the IBB-PCSC and SBB-SCSC equalizers, which achieves the $\mathrm{AC} 2 \mathrm{AC}$ equalization between the two-cell groups, speeding up the balance process with similar balancing current. However, the disadvantage is that this equalizer is only applied to even numbers of cells because of the BB converter for two cells.

The proposed CBB-PCSC equalizer combines the advantages of the IBB-PCSC and SBB-PCSC equalizers. It is easy to extend as it is modular but also has medium cost but small size. Particularly, the balancing speed is the fastest because the $\mathrm{AC} 2 \mathrm{AC}$ equalizations can be achieved both within and among the two-cell groups, further resulting in a highest balancing efficiency (96\%). However, the effectiveness of the CBB is utterly dependent on the consistency of the multiple windings of the transformer. In addition, the cost $(\$ 136)$ is higher than the SBB-SCSC equalizer and SBB-PCSC equalizer (both $13.3 \%$ increase) due to the multi-winding transformers.

\section{CONCLUSION}

In this paper, an attempt has been made to design integrated equalizers based on joint $\mathrm{BB}$ and $\mathrm{SC}$ converters without increasing the quantity of the MOSFETs and drivers. Through detailed experimental analyses for four and six seriesconnected $\mathrm{Li}$-ion batteries and the comparisons with several existed topologies, some conclusions can be obtained as follows, 1) By integrating the $\mathrm{BB}$ converter and $\mathrm{SC}$ converter into one topology, the three proposed equalizers present their own benefits, which is beneficial for the future improvement of integrated equalization topology. 2) With $\mathrm{AC} 2 \mathrm{AC}$ equalization mode, the proposed IBB-PCSC, SBB-PCSC and CBB-PCSC equalizers all present quicker speed and higher efficiency than those of BB equalizer. 3) The IBB-PCSC equalizer is a basic integrated topology, which can be easily scaled for odd and even cell numbers. 4) The proposed SBB-PCSC equalizer presents faster balancing speed and efficiency than those of SBB-SCSC equalizer [19] due to the ability of achieving $\mathrm{AC} 2 \mathrm{AC}$ equalization through PCSC part. 5) Because the dual $\mathrm{AC} 2 \mathrm{AC}$ equalizations are achieved through both $\mathrm{CBB}$ and PCSC parts, the proposed CBB-PCSC equalizer owns the highest speed and efficiency with compact size and acceptable cost, becoming the most efficient one for the series-connected battery string in this study.

\section{REFERENCES}

[1] Y. Shang, K. Liu, N. Cui, N. Wang, K. Li, and C. Zhang, "A compact resonant switched-capacitor heater for lithium-ion battery self-heating at low temperatures," IEEE Transactions on Power Electronics, vol. 35, no. 7, pp. 7134-7144, jul 2020.

[2] X. Tang, C. Zou, K. Yao, G. Chen, B. Liu, Z. He, and F. Gao, "A fast estimation algorithm for lithium-ion battery state of health," Journal of Power Sources, vol. 396, pp. 453-458, aug 2018.

[3] K. Liu, C. Zou, K. Li, and T. Wik, "Charging pattern optimization for lithium-ion batteries with an electrothermal-aging model," IEEE Transactions on Industrial Informatics, vol. 14, no. 12, pp. 5463-5474, dec 2018.

[4] X. Tang, K. Liu, X. Wang, F. Gao, J. Macro, and W. D. Widanage, "Model migration neural network for predicting battery aging trajectories," IEEE Transactions on Transportation Electrification, pp. 1-1, 2020.

[5] H. Chen, L. Zhang, and Y. Han, "System-theoretic analysis of a class of battery equalization systems: Mathematical modeling and performance evaluation," IEEE Transactions on Vehicular Technology, vol. 64, no. 4, pp. 1445-1457, apr 2015.

[6] K. Liu, Y. Li, X. Hu, M. Lucu, and W. D. Widanage, "Gaussian process regression with automatic relevance determination kernel for calendar aging prediction of lithium-ion batteries," IEEE Transactions on Industrial Informatics, vol. 16, no. 6, pp. 3767-3777, jun 2020.

[7] Q. Ouyang, Z. Wang, K. Liu, G. Xu, and Y. Li, "Optimal charging control for lithium-ion battery packs: A distributed average tracking approach," IEEE Transactions on Industrial Informatics, vol. 16, no. 5, pp. 3430-3438, may 2020.

[8] K. Liu, X. Hu, Z. Wei, Y. Li, and Y. Jiang, "Modified gaussian process regression models for cyclic capacity prediction of lithium-ion batteries," IEEE Transactions on Transportation Electrification, vol. 5, no. 4, pp. 1225-1236, dec 2019.

[9] Y. Shang, B. Xia, C. Zhang, N. Cui, J. Yang, and C. Mi, "A modularization method for battery equalizers using multiwinding transformers," IEEE Transactions on Vehicular Technology, vol. 66, no. 10, pp. 87108722 , oct 2017.

[10] H. Xiong, Y. Fu, and K. Dong, "A novel point-to-point energy transmission voltage equalizer for series-connected supercapacitors," IEEE Transactions on Vehicular Technology, vol. 65, no. 6, pp. 4669-4675, jun 2016.

[11] M. Uno and A. Kukita, "Single-switch single-transformer cell voltage equalizer based on forward-flyback resonant inverter and voltage multiplier for series-connected energy storage cells," IEEE Transactions on Vehicular Technology, vol. 63, no. 9, pp. 4232-4247, nov 2014.

[12] A. Xu, S. Xie, and X. Liu, "Dynamic voltage equalization for seriesconnected ultracapacitors in EV/HEV applications," IEEE Transactions on Vehicular Technology, vol. 58, no. 8, pp. 3981-3987, oct 2009.

[13] J. Gallardo-Lozano, E. Romero-Cadaval, M. I. Milanes-Montero, and M. A. Guerrero-Martinez, "Battery equalization active methods," Journal of Power Sources, vol. 246, pp. 934-949, jan 2014.

[14] Z. Zhang, H. Gui, D.-J. Gu, Y. Yang, and X. Ren, "A hierarchical active balancing architecture for lithium-ion batteries," IEEE Transactions on Power Electronics, vol. 32, no. 4, pp. 2757-2768, apr 2017.

[15] P. Cassani and S. Williamson, "Feasibility analysis of a novel cell equalizer topology for plug-in hybrid electric vehicle energy-storage systems," IEEE Transactions on Vehicular Technology, vol. 58, no. 8, pp. 3938-3946, oct 2009.

[16] X. Tang, C. Zou, T. Wik, K. Yao, Y. Xia, Y. Wang, D. Yang, and F. Gao, "Run-to-run control for active balancing of lithium iron phosphate 
battery packs," IEEE Transactions on Power Electronics, vol. 35, no. 2, pp. 1499-1512, feb 2020.

[17] T. H. Phung, A. Collet, and J.-C. Crebier, "An optimized topology for next-to-next balancing of series-connected lithium-ion cells," IEEE Transactions on Power Electronics, vol. 29, no. 9, pp. 4603-4613, sep 2014.

[18] C. Pascual and P. Krein, "Switched capacitor system for automatic series battery equalization," in Proceedings of APEC 97 - Applied Power Electronics Conference. IEEE.

[19] X. Lu, W. Qian, and F. Z. Peng, "Modularized buck-boost+cuk converter for high voltage series connected battery cells," in 2012 Twenty-Seventh Annual IEEE Applied Power Electronics Conference and Exposition (APEC). IEEE, feb 2012.

[20] K.-M. Lee, S.-W. Lee, Y.-G. Choi, and B. Kang, "Active balancing of liion battery cells using transformer as energy carrier," IEEE Transactions on Industrial Electronics, vol. 64, no. 2, pp. 1251-1257, feb 2017.

[21] M.-Y. Kim, J.-H. Kim, and G.-W. Moon, "Center-cell concentration structure of a cell-to-cell balancing circuit with a reduced number of switches," IEEE Transactions on Power Electronics, vol. 29, no. 10, pp. 5285-5297, oct 2014.

[22] Y. Chen, X. Liu, Y. Cui, J. Zou, and S. Yang, "A multi-winding transformer cell-to-cell active equalization method for lithium-ion batteries with reduced number of driving circuits," IEEE Transactions on Power Electronics, pp. 1-1, 2015.

[23] S.-H. Park, K.-B. Park, H.-S. Kim, G.-W. Moon, and M.-J. Youn, "Single-magnetic cell-to-cell charge equalization converter with reduced number of transformer windings," IEEE Transactions on Power Electronics, vol. 27, no. 6, pp. 2900-2911, jun 2012.

[24] A. M. Imtiaz and F. H. Khan, “"'Time shared flyback converter" based regenerative cell balancing technique for series connected li-ion battery strings," IEEE Transactions on Power Electronics, vol. 28, no. 12, pp. 5960-5975, dec 2013.

[25] L. McCurlie, M. Preindl, and A. Emadi, "Fast model predictive control for redistributive lithium-ion battery balancing," IEEE Transactions on Industrial Electronics, vol. 64, no. 2, pp. 1350-1357, feb 2017.

[26] C.-S. Lim, K.-J. Lee, N.-J. Ku, D.-S. Hyun, and R.-Y. Kim, “A modularized equalization method based on magnetizing energy for a series-connected lithium-ion battery string," IEEE Transactions on Power Electronics, vol. 29, no. 4, pp. 1791-1799, apr 2014.

[27] C.-H. Kim, M.-Y. Kim, H.-S. Park, and G.-W. Moon, "A modularized two-stage charge equalizer with cell selection switches for seriesconnected lithium-ion battery string in an HEV," IEEE Transactions on Power Electronics, vol. 27, no. 8, pp. 3764-3774, aug 2012.

[28] M. Arias, J. Sebastian, M. M. Hernando, U. Viscarret, and I. Gil, "Practical application of the wave-trap concept in battery-cell equalizers," IEEE Transactions on Power Electronics, vol. 30, no. 10, pp. 56165631, oct 2015.

[29] Y. Ye, K. W. E. Cheng, Y. C. Fong, X. Xue, and J. Lin, "Topology, modeling, and design of switched-capacitor-based cell balancing systems and their balancing exploration," IEEE Transactions on Power Electronics, vol. 32, no. 6, pp. 4444-4454, jun 2017.

[30] Y. Shang, B. Xia, C. Zhang, N. Cui, J. Yang, and C. C. Mi, "An automatic equalizer based on forward-flyback converter for seriesconnected battery strings," IEEE Transactions on Industrial Electronics, vol. 64, no. 7, pp. 5380-5391, jul 2017.

[31] Y. Shang, C. Zhang, N. Cui, and J. M. Guerrero, "A cell-to-cell battery equalizer with zero-current switching and zero-voltage gap based on quasi-resonant lc converter and boost converter," IEEE Transactions on Power Electronics, vol. 30, no. 7, pp. 3731-3747, jul 2015.

[32] Y. Shang, N. Cui, B. Duan, and C. Zhang, "Analysis and optimization of star-structured switched-capacitor equalizers for series-connected battery strings," IEEE Transactions on Power Electronics, vol. 33, no. 11, pp. 9631-9646, 2017.

[33] F. Mestrallet, L. Kerachev, J.-C. Crebier, and A. Collet, "Multiphase interleaved converter for lithium battery active balancing," IEEE Transactions on Power Electronics, vol. 29, no. 6, pp. 2874-2881, jun 2014.

[34] Y. Shang, K. Liu, N. Cui, Q. Zhang, and C. Zhang, "A sine-wave heating circuit for automotive battery self-heating at subzero temperatures," IEEE Transactions on Industrial Informatics, vol. 16, no. 5, pp. 33553365 , may 2020.

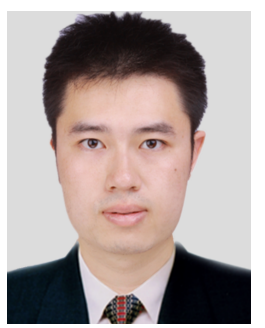

Kailong Liu (M'18) is a Senior Research Fellow in the Warwick Manufacturing Group, University of Warwick, United Kingdom. He received the B.Eng. degree in electrical engineering and the M.Sc. degree in control theory and control engineering from Shanghai University, China, and the Ph.D. degree in electrical engineering from Queen's University Belfast, United Kingdom, in 2011, 2014, and 2018, respectively. His research interests include modeling, optimization and control with applications to electrical/hybrid vehicles, energy storage, battery manufacture and management.

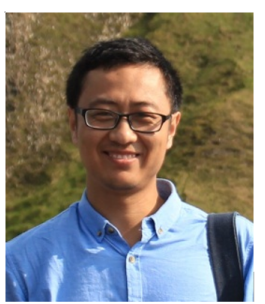

Zhile Yang (S'13, M'17) obtained his BSc in Electrical Engineering and the MSc degree in Control Engineering both from Shanghai University in 2010 and 2013 respectively, and he then received $\mathrm{Ph} . \mathrm{D}$. degree at the School of Electrical, Electronics and Computer Science, Queen's University Belfast, UK. He is currently an associate professor in Shenzhen Institute of Advanced Technology, Chinese Academy of Sciences, Shenzhen, China. His research interests focus on artificial intelligence advanced manufacturing. methods and their applications on smart grid and

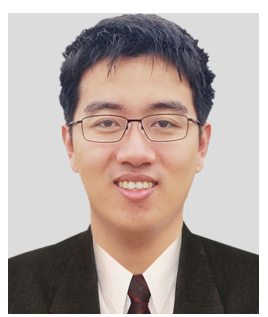

Xiaopeng Tang received the B.S. in automation from the University of Science and Technology of China, He Fei, China, in 2015. He is currently working towards the Ph.D. degree in chemical and biological engineering with the Hong Kong University of Science and Technology, Hong Kong, China. His research interests include modeling and control of energy storage systems.

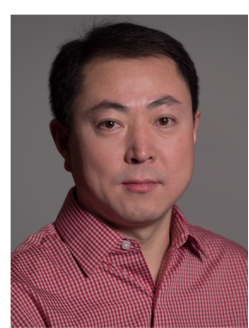

electronics.
Wenping Cao (M'05-SM'11) received the B.Eng in electrical engineering from Beijing Jiaotong University, Beijing, China, in 1991, and the Ph.D. degree in electrical machines and drives from the University of Nottingham, Nottingham, U.K., in 2004. He is currently Chair Professor of Electrical Power Engineering at Aston University, Birmingham, U.K. Prof. Cao is presently the Chairman for the Industrial Electronics Society, IEEE UK and Ireland Section. His research interests include fault analysis and condition monitoring of electrical machines and power 